\title{
DIALED: Data Integrity Attestation for Low-end Embedded Devices
}

\author{
Ivan De Oliveira Nunes \\ University of California, Irvine \\ ivanoliv@uci.edu
}

\author{
Sashidhar Jakkamsetti \\ University of California, Irvine \\ sjakkams@uci.edu
}

\author{
Gene Tsudik \\ University of California, Irvine \\ gene.tsudik@uci.edu
}

\begin{abstract}
Verifying integrity of software execution in low-end microcontroller units (MCUs) is a well-known open problem. The central challenge is how to securely detect software exploits with minimal overhead, since these MCUs are designed for low cost, low energy and small size. Some recent work yielded inexpensive hardware/software co-designs for remotely verifying code and execution integrity. In particular, a means of detecting unauthorized code modifications and control-flow attacks were proposed, referred to as Remote Attestation $(\mathcal{R} A)$ and Control-Flow Attestation (CFA), respectively. Despite this progress, detection of dataonly attacks remains elusive. Such attacks exploit software vulnerabilities to corrupt intermediate computation results stored in data memory, changing neither the program code nor its control flow. Motivated by lack of any current techniques (for low-end MCUs) that detect these attacks, in this paper we propose, implement and evaluate DIALED ${ }^{1}$, the first Data-Flow Attestation (DFA) technique applicable to the most resource-constrained embedded devices (e.g., TI MSP430). DIALED works in tandem with a companion $\mathcal{C}$ FA scheme to detect all (currently known) types of runtime software exploits at fairly low cost.
\end{abstract}

\section{INTRODUCTION}

Embedded systems are growing in number and variety in recent years, rapidly becoming ubiquitous in many aspects of modern society. Some are ultra-cheap and specialized, built atop low-energy, low-cost, and tiny MCUs, e.g., TI MSP430 and AVR ATMega32. Despite being extremely resource-constrained, these MCUs often perform safety-critical tasks that involve sensing and/or actuation. Hence, their security is very important.

Securing low-end MCUs is very challenging, since they often lack any security-relevant hardware features. At the lowest end of the spectrum, devices have no MMUs or MPUs, and thus have no means to support an OS or even a microkernel. Hence, sophisticated malware prevention and detection techniques that work on smartphones, laptops, desktops, and servers are inapplicable. To address this problem, a number of architectures [12], [8], [18], [3], [16], [20], [14], [19], [9] were proposed to support rudimentary security services on low-end MCUs. One prominent research direction involves socalled hybrid (hardware/software co-design) architectures that offer strong security guarantees at minimal hardware cost.

In that vein, architectures for Remote Attestation $(\mathcal{R A})$ [12], [8], [3], Proofs of Execution (PoX) [18], and Control-Flow Attestation [9] are of particular interest. $\mathcal{R A}$ is an interaction between a trusted and more powerful entity, called a Verifier $\left(\mathcal{V}_{\text {rf }}\right)$ and a potentially compromised remote low-end device, called a Prover $(\mathcal{P} r v)$. It allows $\mathcal{V}$ rf to securely measure $\mathcal{P}$ rv's memory contents, enabling detection of malware that modifies code installed on $\mathcal{P}$ rv. PoX augments $\mathcal{R} A$ by providing $\mathcal{V}$ rf with a proof that the attested code was executed and that any claimed outputs (e.g., sensed values) were indeed produced by executing the expected code. Finally, $\mathcal{C} F A$ detects control-flow attacks, whereby benign code on $\mathcal{P}$ rv contains unknown bugs (e.g., buffer overflows caused by lack of array bound checks) that can

\footnotetext{
${ }^{1}$ To appear at DAC'21
}

be exploited to hijack the program's control-flow, i.e., the order of instruction execution. In summary, $\mathcal{C}$ FA provides $\mathcal{V}_{\mathrm{rf}}$ with a proof that benign code was executed in a particular valid or expected order.

Despite these advances, detection of data-only attacks remains elusive. Similar to control-flow attacks, data-only attacks exploit vulnerabilities in benign code to corrupt intermediate values in datamemory. It is well-known [22], [15] that data-only attacks need not alter the code or its control-flow in order to corrupt data. Without a way to detect data-only attacks (as well as code and control-flow modifications) results of Prv's remote computation cannot be trusted.

Very recently, a novel technique supporting both $\mathcal{C} F A$ and $\mathcal{D} F A$, called OAT [21], was proposed. However, it implements $\mathcal{D} F A$ by relying on trusted hardware support from ARM TrustZone, which is only available on higher-end platforms (e.g., smartphones, Raspberry $\mathrm{Pi}$, and similar) and is not affordable to low-end, low-energy MCUs. In addition, OAT's security relies on the application programmer's ability to correctly annotate all critical variables in the code to be attested. This is a strong assumption, since most control-flow and data-only exploits are caused by implementation bugs introduced by the very same application programmer. Naturally, it would be beneficial for this assumption to be avoided.

In this paper, we focus on security against data-only attacks on low-end MCUs and propose DIALED: Data Integrity in Attestation for Low-end Embedded Devices. DIALED's only hardware requirement is that already provided (at relatively low-cost) by the PoX architecture APEX [18]. DIALED uses APEX to securely log and authenticate any data inputs used by the program. This authenticated $\log$ allows $\mathcal{V}$ rf to reconstruct the entire data-flow of the program's execution, thus enabling detection of any data-corruption attacks via abstract execution of the attested program.

Similar to OAT, DIALED is implemented alongside Tiny-CFA [9], a low-cost $\mathcal{C} F A$ technique. This composition enables, for the first time, detection of both control-flow and data-only attacks for lowend MCUs. Notably, DIALED does not rely on code annotation; thus, its security neither requires, nor depends on, any human intervention. In the rest of this paper, we describe DIALED's design and analyze its security. We also report on the implementation of DIALED along with Tiny-CFA on the TI MSP430 MCU and demonstrate its costeffectiveness by providing both $\mathcal{C} F A$ and $\mathcal{D}$ FA for three applications.

\section{Background \& Problem Statement}

This section overviews targeted devices and defines the problem setting.

\section{A. Scope of Low-End MCUs}

This paper focuses on tiny CPS/IoT sensors and actuators, or hybrids thereof. These are some of the smallest and weakest devices based on low-power single-core MCUs with small program and data 
memory (e.g., aforementioned Atmel AVR ATMega and TI MSP430), with 8 - and 16 -bit CPUs running at $1-16 \mathrm{MHz}$, with $\approx 64$ KBytes of addressable memory. SRAM is used as data memory, normally ranging between 4 and 16KBytes, while the rest of the address space is available for program memory. Such devices usually run software atop "bare metal", execute instructions in place (physically from program memory), and lack any memory management unit (MMU) to support virtual memory.

\section{B. Control-Flow vs. Data-Only Attacks}

Both control-flow and data-only attacks violate program execution integrity without modifying the actual executable, by taking advantage of implementation bugs, e.g., lack of array bound checks. Such vulnerabilities are quite common in memory-unsafe languages, such as $\mathrm{C}, \mathrm{C}++$, and Assembly, which are widely used to program MCUs.

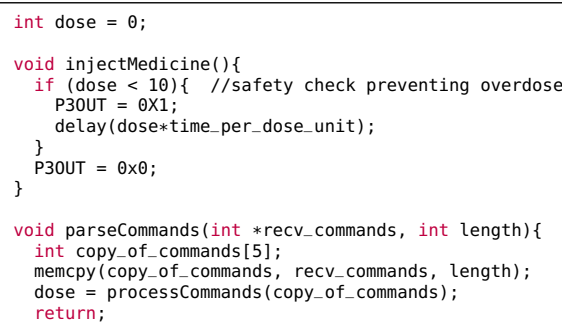

Fig. 1. Embedded application vulnerable to a control-flow attack [9].

Control-flow attacks change the order of instructions execution thus changing program behavior, escalating privilege, and/or bypassing safety checks. Consider the example in Figure 1 (taken from [9]). In this embedded application, the MCU is connected through the general purpose input/output (GPIO) port P3OUT (lines 5 and 8) to an actuator that injects a certain dose of medicine, determined in software, according to commands received from the network, e.g., from a remote physician.

The function injectMedicine injects appropriate dosage given by the variable dose, by triggering actuation for period of time proportional to the value of dose. To guarantee a safe dosage, the if statement (line 4) assures that the maximum injected dosage is 9 , thus preventing the patient from over-dosing due to errors. The function parseCommands (line 11) makes a copy of received commands and processes them to determine the appropriate dosage. However, this function can be abused by a control-flow attack at line 13. Specifically, because copy_of_commands has a fixed length of 5 , an input array of size more than 5 causes a buffer overflow corrupting the data in stack, including the return address of parseCommands. In particular, the return address can be overwritten with the value of recv_commands [5]. By setting the content of parseCommands [5] to be the address of line 5, such an attack causes the control flow to jump directly to line 5 , skipping the safety check at line 4 , and potentially overdosing the patient.

As mentioned earlier, $\mathcal{C}$ FA securely logs all control-flow deviations and (upon request) provides an authenticated copy of this log to $\mathcal{V}$ rf, enabling detection of the aforementioned attack. We overview a concrete $\mathcal{C}$ FA architecture in Section II-C. However, $\mathcal{C}$ FA cannot detect data-only attacks that do not change the control-flow. Figure 2 presents a second implementation attempt, vulnerable to data-only attacks. To see this vulnerability, note that P3OUT register controls multiple physical ports, each associated with one bit in P3OUT register, e.g.:
- Setting P3OUT $=00000000=0 \times 0$ turns all physical ports off

- Setting P3OUT $=00000001=0 \times 1$ turns on 1st physical port

- Setting P3OUT $=00000010=0 \times 2$ turns on 2 nd physical port

- Setting P3OUT $=00000011=0 \times 3$ turns on both 1 st and 2 nd physical ports, etc.

In order to trigger actuation through the proper port (Port 1 in this example), this code needs to set P3OUT $=0 \times 1$. This is configured in the global variable set, at line 1 of Figure 2 . The value of set is later used to trigger actuation at line 8 . The code also allows settings to be updated at an arbitrary position defined by the input parameter index. Since settings has a fixed length of 8 , a malicious input with index $=8$ would overflow this buffer causing set to be overwritten with the value of new_setting. An input new_setting $=0$ with index $=9$ would overwrite set $=0$. Later, at line 8, when set is used to trigger actuation of port 1 , it will instead have no actuation effect (since it is now 0). Consequently, the medicine will not be injected. It is important to note that this attack does not change the program control flow, but just corrupts data. It therefore can not be detected by $\mathcal{C F A}$ alone.

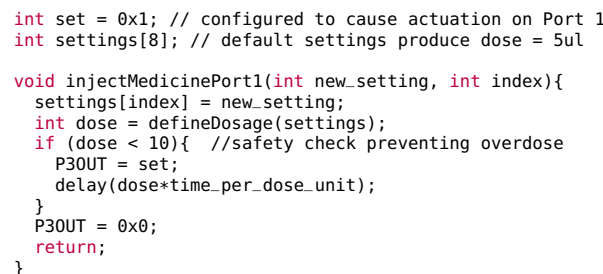

Fig. 2. Embedded application vulnerable to a data-flow attack.

\section{Prior Work: CFA}

In recent years several $\mathcal{C} F A$ techniques have been proposed [2], [11], [10], [23], [21]. We focus on Tiny-CFA, the only $\mathcal{C} F A$ for low-end devices targeted in this work. Tiny-CFA is constructed atop another recently proposed technique - APEX formally verified architecture [18] that implements the so-called Proof-of-Execution (PoX) primitive. Tiny-CFA imposes no additional hardware requirements, beyond those imposed by APEX. The PoX primitive allows $\mathcal{P} r v$ to prove to $\mathcal{V} r$ cryptographically binding the following guarantees:

1) The proper/expected code is loaded at a particular location in $\mathcal{P}$ rv's program memory, referred to as Executable Range (ER).

2) This proper/expected code was executed in its entirety: from its first instruction $=$ its legal entry point, until its last instruction $=$ its legal exit.

3) All outputs are authentic and were indeed produced by this execution. The outputs are written to a specific memory location, referred to as Output Range (OR).

Sizes and locations of ER and OR are configurable, which enables PoX for arbitrary code and arbitrary outputs. APEX is formally verified to guarantee secure PoX even in case of a full software compromise of the underlying MCU. APEX also prevents any external data memory modifications that could attempt to tamper with ER's state while the code in ER is running (e.g., via interrupts or DMA).

Tiny-CFA implements $\mathcal{C} F A$ atop APEX by instrumenting the executable with instructions that log the control flow to APEXauthenticated OR region. Specifically, given an executable, TinyCFA instruments all control flow-altering instructions (e.g., jumps, branches, function calls, and returns) by logging the destination address of each such instruction to the OR region. Tiny-CFA also assures that the $\log$ in $\mathrm{OR}$ cannot be modified. This way, given 


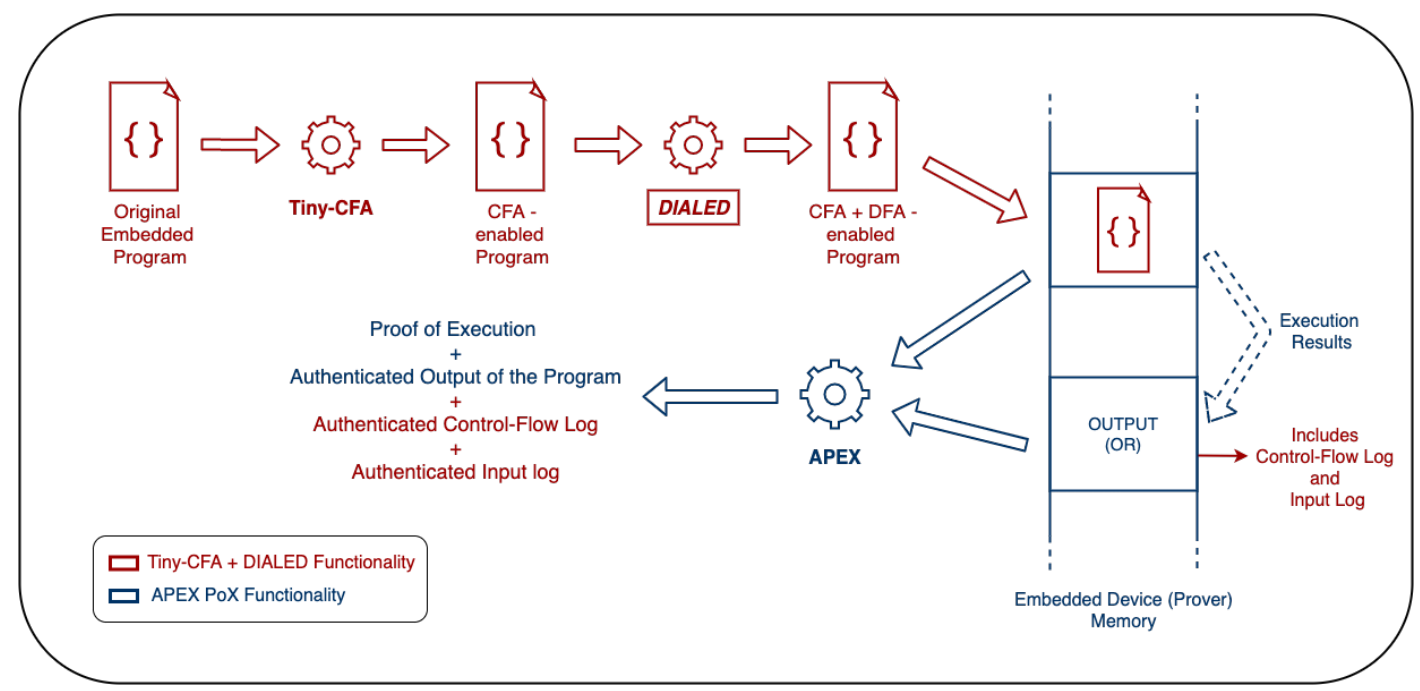

Fig. 3. DIALED Architectural Components.

APEX formal guarantees, $\mathcal{V}_{\mathrm{rf}}$ securely learns the exact control flow of program execution on a remote $\mathcal{P}$ rv.

DIALED also uses code instrumentation and augments Tiny-CFA with the detection of data-only attacks. DIALED's core idea is to $\log$ (and send to $\mathcal{V}$ rf) all the inputs of the program during execution along with its control flow, which enables $\mathcal{V}$ rf to emulate the entire execution of the attested program, allowing it to detect both controlflow and data-flow attacks. DIALED is detailed in the next section.

\section{DIALED DESIGN}

Figure 3 shows the components of DIALED: it is implemented alongside Tiny-CFA (itself based on APEX) to provide both $\mathcal{C}$ FA and $\mathcal{D} F A$. The executable is separately instrumented by both TinyCFA and DIALED. APEX provides a proof of the execution of the instrumented executable, serving as an authenticator for its output: a log containing the executable's control flow and its data inputs.

\section{A. Overview}

To detect data-only attacks, DIALED uses a novel input detection method via secure instrumentation of the executable. This instrumentation guarantees that all relevant data is logged during program execution; this is in addition to the control-flow log produced by Tiny-CFA. The underlying PoX primitive provides $\mathcal{V} r f$ with a proof that this output (containing all relevant data and the control-flow path) was indeed produced by the execution of the expected (instrumented) code. In doing so, DIALED provides $\mathcal{V}_{\mathrm{rf}}$ with all information needed to abstractly execute this program locally and detect any code, control flow, or data compromises. The core feature of DIALED is detection and secure logging of every external input received during program execution, including input from peripherals, the network, GPIO, as well as data fetches from memory locations outside the executable's own state.

Similar to OAT [21], the goal is to attest embedded operations, i.e., finite and self-contained safety-critical functions called by the program's main loop. Examples include sensing and actuation tasks triggered by commands received through the network, as in Section II-B. Since embedded operations typically have well defined and reasonably small number of data inputs, DIALED can efficiently save all inputs to an append-only log - Input Log (I-Log). DIALED instrumentation assures that all data inputs are appended to I-Log.
Definition 1 (Data Inputs). Any value read from any memory location outside of the attested program's current stack. The program's current stack is the region located within the current stack pointer value (top of the stack) and the value of the stack pointer when the attested program was first called (based of the program's stack). It includes all local variables.

According to this definition, read instructions that move/copy data from peripherals, network or GPIO are considered as Data Inputs and written to I-Log, since these involve reads from memory outside of the program's stack. But reads that occur during regular computation, e.g., instructions which compute on local variables are not written to I-Log, as they are not inputs. This approach makes the size of ILog relatively small, which is confirmed by the real-world embedded operations considered in our evaluation in Section V.

Recall that Tiny-CFA instruments the executable to produce a Control-Flow Log (CF-Log). In DIALED, both CF-Log and I-Log are written to APEX-designated output region $O R$. Hence, $\mathcal{V} r f$ is assured of the integrity of these logs. In addition, given the attestation guarantee, $\mathcal{V}$ rf is also assured that the correct/expected instrumented code was executed to produce this log. By knowing the code, its control-flow, and all inputs, $\mathcal{V}$ rf can locally emulate its execution and verify all steps in this computation, as well as detect all data-only and control-flow attacks.

\section{B. Adversary Model}

We assume an adversary that controls Prv's entire software state, including code and data. It can modify any writable memory and read any memory that is not explicitly protected by hardware-enforced access controls, e.g., APEX rules. Program memory modifications can change instructions, while data memory modifications can trigger control-flow and data-only attacks arbitrarily. Adversarial modification attempts are allowed before, during, or after the execution.

\section{Design Rationale}

DIALED's security is based on five features: F1-F5. We describe them at a high level in this section and discuss how to realize an instance of DIALED on MSP430 via automated code instrumentation in Section IV.

(F1) Integrity Proofs for Code, Instrumentation, and Output 
As an instrumentation-based technique, DIALED is only secure if any modifications to the instrumented code itself (e.g., removing instrumented instructions) is detectable. Detection of code modifications is already offered by the underlying APEX PoX architecture (see Section II-C). APEX guarantees that every code modification is detected by $\mathcal{V} r$. It also guarantees that any modification of the attested executable's output region $O R$ (which, in our case, includes CF-Log and I-Log) can only be done by the attested executable itself, during its execution.

\section{(F2) Integrity Proof for the Control Flow}

Since DIALED relies on instrumented instructions, these instructions can not be skipped, e.g., via control-flow violations. Therefore, Tiny-CFA ensures that the control flow is logged to CF-Log and whatever is written to CF-Log can not be modified; see [9] for details. Hence, all attempts to skip the logging of any data inputs are detectable by $\mathcal{V}$ rf using CF-Log. The integrity of CF-Log itself is important to DIALED's overall functionality, since $\mathcal{V} r f$ needs both CF-Log and I-Log in order to abstractly execute the program and verify the integrity of the execution.

\section{(F3) Secure Logging of Data Inputs from Operation Arguments}

To enable abstract execution by $\mathcal{V}$ rf, any arguments passed to the program at invocation must be securely logged to I-Log. DIALED automatically instruments the executable with Assembly instructions that copy all program arguments to I-Log.

\section{(F4) Secure Logging of Runtime Data Inputs}

In addition to arguments, data inputs can be obtained at runtime, e.g., sensed values read from GPIO, or packets arriving from the network. Such inputs are received through peripheral memory, at a particular set of physical addresses in data-memory. To detect and log these inputs DIALED instruments every read instruction to check whether the read address is outside the program's stack. The range of the stack is determined by $[l s, h s]$, where $l s$ is the value of the stack pointer saved at the moment when execution starts (before the allocation of local variables), and $h s$ always reflects the current stack pointer, i.e., the top of the stack.

\section{(F5) I-Log and CF-Log Integrity}

To ensure integrity of CF-Log and I-Log, DIALED must guarantee that control flow and data-only attacks do not overwrite these logs. Thus, we realize I-Log and CF-Log as a single stack data structure inside $\mathrm{OR}$, from the highest value $\left(O R_{\max }\right)$ growing downwards. The pointer to the top of this stack is stored in a dedicated register $\mathcal{R}$. Each instruction that alters the control flow or involves data input is instrumented (with additional instructions) to push the relevant values (either control-flow destination or data input) onto the stack, i.e.:

1) Write the value (destination of address or data input) to the location pointed by $\mathcal{R}$; and

2) Decrement $\mathcal{R}$.

At instrumentation time, assembly code is inspected to ensure that no other instructions use $\mathcal{R}$. In all practical code examples we inspected, executables have at least one free register available. If no such register exists, the code can be recompiled to free up one register. Whenever a write operation occurs, it is checked for safety, by seeing if the address of the write is within the range $\left[\mathcal{R}, O R_{\max }\right]$, i.e., the current range for I-Log and CF-Log. If an illegal write occurs, execution is aborted and $\mathcal{V}$ rf treats it as an attack. Since these "write checks" are already needed, and implemented, by Tiny-CFA, they can be used "as is" by DIALED, at no additional instrumentation cost.

\section{Security Analysis}

Let $\mathcal{O} p$ denote an embedded operation for which control-flow and data-flow need to be attested. Feature $\mathbf{F 1}$ assures to $\mathcal{V}$ rf that $\mathcal{O} p$ indeed executed, and that neither its executable (including instructions added by DIALED's instrumentation) nor the output (OR) produced by this execution has been tampered with. Feature $\mathbf{F 2}$ assures that all changes to the control-flow of $\mathcal{O} p$ are written to $O R$ at runtime. Similarly, F3 \& F4 guarantee that any data inputs are also logged to $O R$. Therefore, what we need to show is that, once written, controlflow and data input values in $O R$ can not be modified during the rest of $\mathcal{O} p$ execution. This is exactly the guarantee offered by $\mathbf{F 5}$. Therefore, DIALED features F1-F5 suffice to guarantee the integrity of $O R$ and $\mathcal{O} p$ 's executable (stored in $E R$ ), including I-Log and CF-Log, even in the presence of potential control-flow and data-only attacks. Given the integrity of received I-Log and CF-Log, $\mathcal{V}$ rf can emulate execution of $\mathcal{O} p$ locally and reproduce any type of runtime attack that may have occurred during $\mathcal{O} p$ 's actual execution in $\mathcal{P}$ rv.

\section{DIALED IMPLEMENTATION}

As described in Section III-C, features F1, F2 and F5 are provided by APEX and Tiny-CFA. Hence, we focus on the implementation of F3-F4 achieved via automated instrumentation of the executable. Our instrumentation component was coded in about 300 lines of Python. In the rest of this section we use $\mathcal{O} p$ to refer to the executable to be instrumented and later attested.

Figure 4 shows the instrumentation used to implement F3 (in MSP430 Assembly) which commits $\mathcal{O} p$ 's arguments to I-Log. The instrumentation is added once: at the entry point of $\mathcal{O} p$ to $\log$ any input parameters. Lines 2-4 are already added to $\mathcal{O} p$ by Tiny-CFA to check whether $\mathcal{R}$ is initialized to $O R_{-} M A X$. This is required by property $\mathbf{F 5}$ (see Section III-C). Lines 5-9 are added by DIALED to save the current stack pointer value to address $O R \_M A X$. This value determines the bottom of $\mathcal{O} p$ 's execution stack and is used to detect and $\log$ data inputs. Lines 10-25 record $\mathcal{O} p$ 's arguments (input parameters) to I-Log. In MSP430, function arguments are passed using up to 8 general-purposes registers $r 8-r 15$. Since the application defines how many arguments are passed, DIALED always logs all of such registers, to guarantee that all inputs are always captured. In this implementation, $\mathcal{R}=r 4$. Hence, each register is written to the memory address pointed by $r 4$. At each such write, safety checks discussed in $\mathbf{F 5}$ (Section III-C) are performed to assure the integrity of I-Log and CF-Log in $O R$. Additional checks are performed to guarantee that $\mathcal{R}=r 4$ never overflows the size of $O R$. Such an event is treated as a security violation and reported to $\mathcal{V}$ rf.

Figure 5 depicts the instrumentation used to log runtime data inputs to I-Log-i.e., feature F4. Line 2 is a read instruction to copy contents from address pointed to by $r 15$, to $r 14$. In order to define whether this is indeed a data input, at line 4 , the address in $r 15$ is checked against the location of the bottom of $\mathcal{O} p$ 's stack, which is stored at the address of $O R \_M A X$ when $\mathcal{O} p$ is invoked (lines 6-9 in Figure 4). Also, at line 6 in Figure 5, the address in $r 15$ is also checked against the current stack pointer (always stored at register $r 1$ ). If these checks fail, the value of the address pointed to by $r 15$ lies outside of $\mathcal{O} p$ 's current execution stack: it is treated as input and committed to ILog at line 9. Otherwise, the value is is part of $\mathcal{O} p$ 's current state and is not logged. Lines 10-12 check if $r 4$ reached the top of $O R$, preventing overflows, as described in the previous paragraph. 


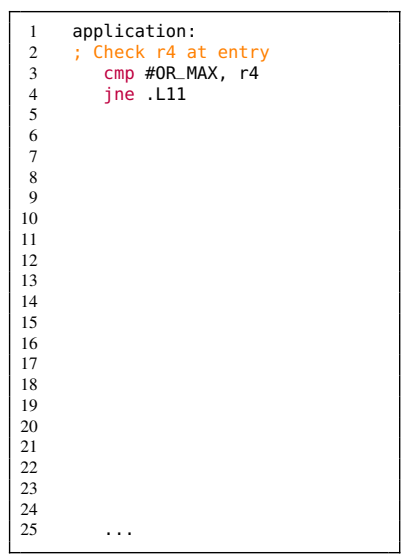

(a) Before DIALED instrumentation

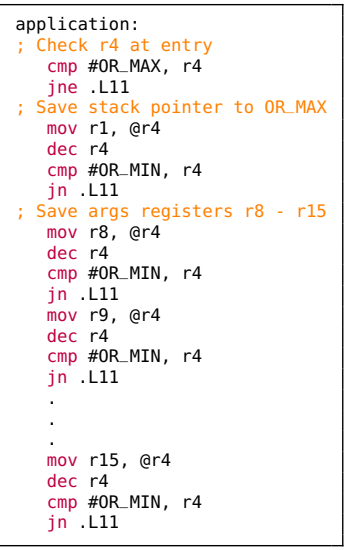

(b) After DIALED instrumentation
Fig. 4. Instrumentation example: Logging $\mathcal{O} p$ 's arguments.

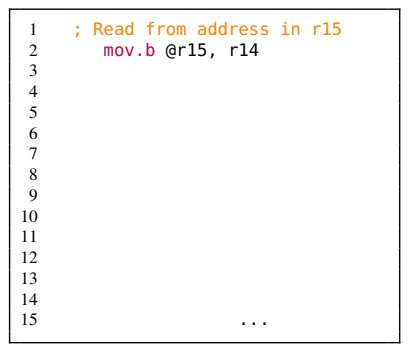

(a) Before DIALED instrumentation

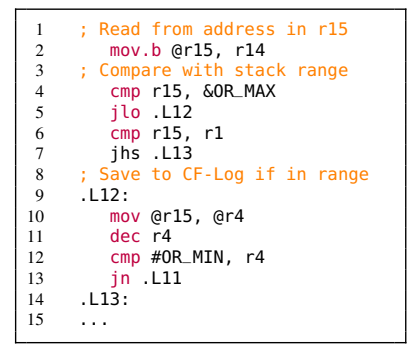

(b) After DIALED instrumentation
Fig. 5. Instrumentation example: Logging runtime data inputs.

Note that, since DIALED is implemented alongside Tiny-CFA, it cannot be abused by control flow attacks that jump in the middle of the instrumented code to skip checks and/or data input logging. Such an illegal jump is itself a control-flow change, which is committed to CF-Log by Tiny-CFA and thus detected by $\mathcal{V}$ rf.

\section{Evaluation}

We evaluate DIALED in terms of its hardware costs and software runtime overhead of attested embedded operations.

\section{A. Hardware Overhead}

Table I compares DIALED functionality and hardware costs to prior runtime attestation techniques (overviewed in Section VI). In terms of hardware, both C-FLAT [2] and OAT [21] are based on ARM TrustZone [4] which is inapplicable to low-end MCUs. Atrium [23], LO-FAT [11] and LiteHAX [10] rely on dedicated hardware support from hash engines and branch-monitoring modules. Thus, their hardware overhead is far more costly than the baseline MCU (MSP430) itself. Meanwhile, DIALED and Tiny-CFA rely exclusively on lowcost hardware support of the APEX's PoX architecture [18]. Thus, they impose much lower hardware overhead, affordable even for such low-end MCUs. Out of all other architectures, only OAT, LiteHAX and DIALED provide both $\mathcal{C} F A$ and DFA. Among these, DIALED achieves $\approx 5 \times$ lower overhead in terms of combinatorial logic (LookUp Tables - LUTs) and $\approx 50 \times$ lower state hardware overhead (Registers) than the cheapest prior technique achieving both $\mathcal{C} F A$ and DFA, i.e., LiteHAX.

\section{B. Experimental Analysis on Real-world Applications}

We evaluate DIALED runtime overhead in three real-world applications. For the sake of fair comparison, we consider the exact

\begin{tabular}{|c||c|c|c|c||}
\hline Technique & $\begin{array}{c}\text { Support } \\
\text { for CFA }\end{array}$ & $\begin{array}{c}\text { Support } \\
\text { for } \mathcal{D} F A\end{array}$ & $\begin{array}{c}\text { Hardware } \\
\text { Cost }- \\
\text { LUTs }\end{array}$ & $\begin{array}{c}\text { Hardware } \\
\text { Cost }- \\
\text { Resigters }\end{array}$ \\
\hline $\begin{array}{c}\text { MSP430 } \\
\text { (baseline) }\end{array}$ & - & - & 1904 & 691 \\
\hline C-FLAT & $\checkmark$ & - & $\begin{array}{c}\text { ARM- } \\
\text { TrustZone }\end{array}$ & $\begin{array}{c}\text { ARM- } \\
\text { TrustZone }\end{array}$ \\
\hline OAT & $\checkmark$ & - & $\begin{array}{c}\text { ARM- } \\
\text { TrustZone }\end{array}$ & $\begin{array}{c}\text { ARM- } \\
\text { TrustZone }\end{array}$ \\
\hline Atrium & $\checkmark$ & - & $\begin{array}{c}10640 \\
(+559 \%)\end{array}$ & $\begin{array}{c}15960 \\
(+2308 \%)\end{array}$ \\
\hline LO-FAT & $\checkmark$ & $\checkmark$ & $\begin{array}{c}3192 \\
(+168 \%)\end{array}$ & $\begin{array}{c}4256 \\
(+616 \%)\end{array}$ \\
\hline LiteHAX & $\checkmark$ & - & $\begin{array}{c}302 \\
(+16 \%)\end{array}$ & $\begin{array}{c}2128 \\
(+308 \%)\end{array}$ \\
\hline Tiny-CFA & $\checkmark$ & $\checkmark$ & $\begin{array}{c}\mathbf{3 0 2} \\
(+\mathbf{1 6 \%})\end{array}$ & $\begin{array}{c}\mathbf{4 4} \\
(+\mathbf{+ 6 \%} \%)\end{array}$ \\
\hline DIALED & $\checkmark$ & & &
\end{tabular}

TABLE I

FUNCTIONALITY AND HARDWARE OVERHEAD COMPARISON OF EXISTING RUN-TIME ATTESTATION ARCHITECTURES

same open-source applications used to evaluate Tiny-CFA: (1) OpenSyringePumpe ${ }^{2}$ - a medical syringe pump; (2) FireSensor ${ }^{3}$; and (3) UltrasonicRanger ${ }^{4}$ - a sensor used in vehicles to measure distance from obstacles.

We consider all sources of runtime overhead imposed by code instrumentation in these techniques: code size increase, runtime (CPU cycles), and the size of the attestation log inside $O R$, including ILog and CF-Log. Figures 6(a) and 6(b) compare results unmodified applications, with the same applications instrumented by Tiny-CFA (CFA guarantee only), and the same applications instrumented by DIALED (both $\mathcal{C} F A$ and DFA guarantees). As these results demonstrate, the overhead in both cases is dominated by the instrumentation required for $\mathcal{C} F A$. On top of Tiny-CFA, DIALED code size and runtime increases range between $1 \%-20 \%$. This is due to additional instructions introduced by DIALED instrumentation, as described in Section IV. Figure 6(c) shows the total $O R$ size required to store the execution information. Recall that DIALED requires storage of both I-Log and CF-Log to enable detection of both control-flow and dataonly attacks. Size requirement for these logs vary widely depending on the type of application (control-flow- or data-input-intensive). In general, we observe a small increase in $O R$ size. This is due to the data input definition from Section III-C, which allows DIALED to only log relevant data inputs while retaining all necessary information for $\mathcal{V}$ rf's abstract execution of $\mathcal{P}$ rv's embedded operation.

Remark: we do not compare runtime overhead of DIALED with $\mathcal{D} F A$ architectures LiteHAX and OAT, since these techniques rely on specific hardware support implemented in different CPU architectures (generally higher-end platforms), with different applications.

In summary, even though DIALED's overhead is certainly not negligible, it is well within the capabilities of low-end MCUs and suitable for practical purposes. Specifically, instrumented binary sizes are within the MCUs memory budget, its runtime is reasonable, and $\log$ sizes are small enough to fit into data memory without encroaching on the stack. We believe this to be a reasonable price for the benefit of detecting any runtime compromise in low-end MCUs.

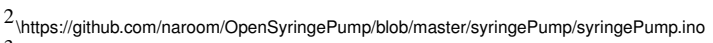

3 https://github.com/Seeed-Studio/LaunchPad_Kit/tree/master/Grove_Modules/temp_humi_sensor

${ }^{4}$ https://github.com/Seeed-Studio/LaunchPad_Kit/tree/master/Grove_Modules/ultrasonic_ranger
} 


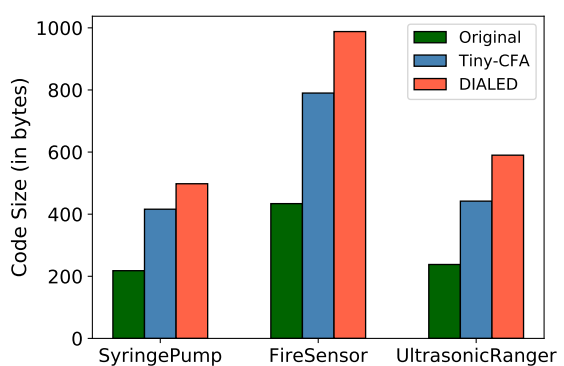

(a) Total code size

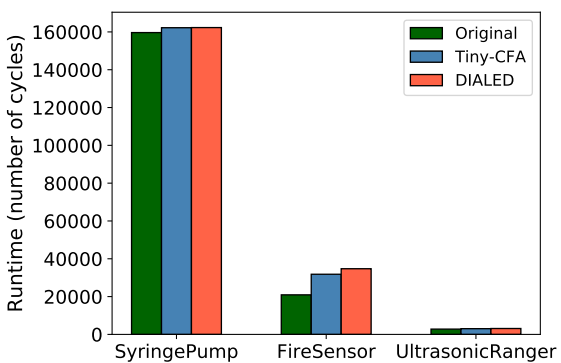

(b) Runtime

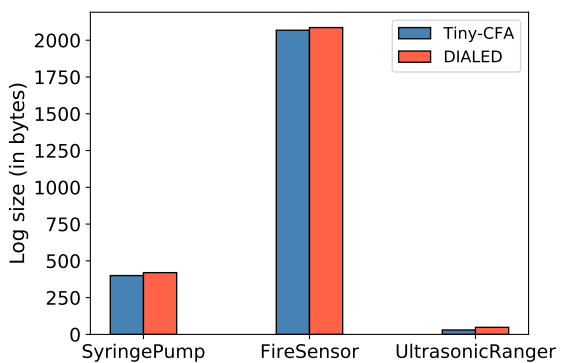

(c) Log size

Fig. 6. Comparison of embedded operation runtime costs: unmodified operation, Tiny-CFA (CFA only), and DIALED (CFA $+\mathcal{D F A})$.

\section{RELATED WORK}

Control-Flow and Data-Flow Integrity [1], [7] are techniques for prevention or detection of data and control-flow corruptions in realtime, locally at $\mathcal{P}$ rv, in contrast with after-the-fact detection by $\mathcal{V}$ rf. All such techniques depend on complex software support (e.g., highend operating systems) and/or heavyweight address-based and valuebased integrity checks at runtime. Unfortunately, all these options are inapplicable to simple MCUs.

(Static) Remote Attestation ( $\mathcal{R A}$ ) [12], [8], [3], [13], [6], [17] is used by $\mathcal{V}_{r f}$ to check if a remote $\mathcal{P} r v$ has the proper software. By itself, static $\mathcal{R A}$ does not provide any runtime guarantees. However, it is used as a building block for most runtime attestation techniques, including $\mathcal{C} F A$ and DFA. DIALED itself is build atop APEX PoX functionality. In turn, APEX relies on VRASED [8] - a formally verified static $\mathcal{R A}$ architecture - to implement PoX.

Runtime Attestation includes techniques such as $\mathcal{C} F A$ and $\mathcal{D} F A$. C-FLAT [2] is the earliest $\mathcal{C} F A$ architecture that uses ARM TrustZone Secure World [5] to implement $\mathcal{C} F A$, by instrumenting the executable with context switches between TrustZone Normal and Secure worlds. At each control flow altering instruction, execution is trapped into Secure World and the control flow path is logged to protected memory. To remove TrustZone dependence, LO-FAT [11] and LiteHAX [10] implement CFA using stand-alone hardware modules: a branch monitor and a hash engine. Atrium [23] enhances aforementioned CFA techniques by securing them against physical adversaries that intercept instructions as they are fetched to the CPU. Though less expensive than C-FLAT, such hardware components are still not affordable for low-end MCUs, since their cost (in terms of price, size, and energy consumption) is higher than that of a lowend MCU itself. OAT [21] and LiteHax [10] also provide DFA (in addition to $\mathcal{C F A}$ ). However, similar to aforementioned techniques, they are too costly for low-end MCUs.

\section{CONCLUSIONS}

We design and implement DIALED, the first Data-Flow Attestation (DFA) approach targeting lowest-end MCUs. DIALED is composed with Tiny-CFA, a Control-Flow Attestation (CFA) architecture, thus enabling detection of both control-flow and data-flow attacks at runtime. We discuss DIALED's security and evaluate its performance on real embedded applications, showing that DIALED's overhead is well within the capabilities of some of the most resource-constrained MCUs.

Acknowledgments: We thank DAC'21 anonymous referees for their helpful comments. This research was supported in part by funding from Army Research Office (ARO) contract W911NF-16-1-0536, Semiconductor Research Corporation (SRC) contract 2019-TS-2907, as well as NSF Awards 1956393 (SATC) and 1840197 (CICI).

\section{REFERENCES}

[1] M. Abadi et al., "Control-flow integrity principles, implementations, and applications," ACM Transactions on Information and System Security (TISSEC), vol. 13, no. 1, pp. 1-40, 2009.

[2] T. Abera et al., "C-flat: Control-flow attestation for embedded systems software," in ACM CCS, 2016.

[3] M. Ammar et al., "Simple: A remote attestation approach for resourceconstrained iot devices," in ACM/IEEE ICCPS, 2020, pp. 247-258.

[4] ARM Security Technology - Building a Secure System using TrustZone Technology, ARM Limited, 2009.

[5] Arm Ltd., "Arm TrustZone," 2018. [Online]. Available: https: //www.arm.com/products/security-on-arm/trustzone

[6] F. Brasser et al., "Tytan: Tiny trust anchor for tiny devices," in DAC. ACM, 2015.

[7] M. Castro et al., "Securing software by enforcing data-flow integrity," in Proceedings of the 7th symposium on Operating systems design and implementation, 2006, pp. 147-160.

[8] I. De Oliveira Nunes et al., "VRASED: A verified hardware/software co-design for remote attestation," USENIX Security'19, 2019.

[9] I. De Oliveria Nunes et al., "Tiny-cfa: A minimalistic approach for control flow attestation using verified proofs of execution." 2021.

[10] G. Dessouky et al., "Litehax: Lightweight hardware-assisted attestation of program execution," in IEEE/ACM ICCAD, 2018.

[11] G. Dessouky et al., "Lo-fat: Low-overhead control flow attestation in hardware," in $D A C, 2017$.

[12] K. Eldefrawy et al., "Smart: Secure and minimal architecture for (establishing dynamic) root of trust," in NDSS. The Internet Society, 2012.

[13] K. Eldefrawy et al., "Hydra: Hybrid design for remote attestation (using a formally verified microkernel)," in Wisec. ACM, 2017.

[14] A. S. et al., "Pioneer: verifying code integrity and enforcing untampered code execution on legacy systems," in ACM SOSP, 2005.

[15] K. K. Ispoglou et al., "Block oriented programming: Automating dataonly attacks," in Proceedings of the 2018 ACM SIGSAC Conference on Computer and Communications Security, 2018, pp. 1868-1882.

[16] R. Kennell et al., "Establishing the genuinity of remote computer systems," in USENIX Security, 2003.

[17] P. Koeberl et al., "TrustLite: A security architecture for tiny embedded devices," in EuroSys. ACM, 2014.

[18] I. D. O. Nunes et al., "APEX: A verified architecture for proofs of execution on remote devices under full software compromise," in 29th USENIX Security Symposium, 2020.

[19] A. Seshadri et al., "SAKE: software attestation for key establishment in sensor networks," in DCOSS, 2008.

[20] A. Seshadri et al., "SWATT: Software-based attestation for embedded devices," in IEEE S\&P '04, 2004.

[21] Z. Sun et al., "Oat: Attesting operation integrity of embedded devices," in IEEE Symposium on Security and Privacy, 2020.

[22] L. Szekeres et al., "Sok: Eternal war in memory," in 2013 IEEE Symposium on Security and Privacy. IEEE, 2013, pp. 48-62.

[23] S. Zeitouni et al., "Atrium: Runtime attestation resilient under memory attacks," in Proceedings of the 36th International Conference on Computer-Aided Design. IEEE Press, 2017, pp. 384-391. 